\title{
Die operative Versorgung der Akromioklavikulargelenkssprengung
}

\author{
Klemens Horst, Thomas Dienstknecht, Hans Christoph Pape
}

\section{Zusammenfassung}

Die Luxation des Akromioklavikulargelenks ist häufig, und eine einheitliche Therapie gibt es nicht. Verletzungen des Gelenks führen zu einer hohen Einbuße an Beweglichkeit. Höhergradige Verletzungen werden typischerweise operativ behandelt. Ein Goldstandard existiert jedoch nicht und mittel- sowie längerfristige Ergebnisse nach Versorgung der Verletzung durch die etablierten Verfahren liegen in guter Qualität nur bedingt bzw. gar nicht vor. Sowohl für die konservative als auch für die operative Versorgung sind z. T. hervorragende Ergebnisse publiziert. Zwei operativ häufig angewandte Verfahren sind die Kirschner-DrahtFixation und die Versorgung mittels TightRope ${ }^{\circledR}$. Diese Verfahren, ihre Indikation sowie Tipps und Tricks werden im vorliegenden Artikel beschrieben. Wir stellen unser Nachbehandlungskonzept der operierten Schulter vor und erörtern die operative Versorgung der AC-Gelenksarthrose.

\section{Surgical Treatment of Acromiocla- vicular Joint Separation}

Dislocations of the acromioclavicular joint are common but there is no uniform therapeutic strategy. Injuries to the joint lead to great restrictions in mobility. Higher degree injuries are typically treated surgically. However, there is no gold standard, also good quality middle- or long-term results of the treatment of such injuries using the established methods are only sparingly, if at all, available. Some, in part, excellent results for not only conservative therapy but also for surgical management have been published. Two frequently used surgical methods are K-wire fixation and management by means of the TightRope ${ }^{\circledR}$. In this article we describe these two processes, their indications and give some tips and tricks. We also present our follow-up concept for the operated shoulder and discuss the surgical management of osteoarthritis of the AC joint.

\section{Einleitung}

Das Akromioklavikulargelenk (ACG) ist Teil des Schulterkomplexes. Degenerative Veränderungen betreffen v. a. ältere Patienten und jene, die vorab eine Verletzung des Gelenks erlitten haben. Eine Luxation ist traumatisch bedingt und betrifft überwiegend junge, sportlich aktive Menschen.

Durch steigende sportliche Aktivität und die Vielfalt der Betätigungsmöglichkeiten ist die Zahl dieser Verletzung in den vergangenen Jahren gestiegen und wird nunmehr regelmäßig beobachtet.

OP-JOURNAL 2013; 29: 24-30

(c) Georg Thieme Verlag KG Stuttgart · New York DOI http://dx.doi.org/10.1055/s-0032-1328234

Schulterverletzungen sind für den Betroffenen mit einer hohen Einbuße an Beweglichkeit verbunden und können bei verspäteter Diagnose oder falscher Behandlung zu erheblichen Spätfolgen führen. ACG-Luxationen sind die dritthäufigste Verletzung des Schultergürtels und machen 4-6\% aller Gelenkluxationen aus [1]. Die Inzidenz variiert je Sportart erheblich und kann bis zu 20\% (Skifahren) betragen. Im Schnitt ist mit 3-4 Fällen pro 100000 Einwohner und Jahr zu rechnen [2].

Eine einheitliche Therapie dieser Verletzungsart gibt es nicht und ist auch heute noch Gegenstand von kontrovers geführten Diskussionen. Für die gewünschte therapeutische Stabilisierung des ACGs stehen mehr als 150 verschiedene Ope- rationsverfahren und konservative Behandlungsmaßnahmen zur Wahl [3].

Mittel- und längerfristige Ergebnisse nach Versorgung der Verletzung durch die etablierten Verfahren liegen in guter Qualität nur bedingt vor, und zu den neueren Operationstechniken liegen keine mittel- bis langfristigen Verlaufskontrollen vor [4-8]. Zurzeit gibt es keinen Goldstandard in der Therapie der ACGLuxation, und in der aktuellen Literatur werden sowohl für die konservative als auch für die operative Versorgung $z$. T. hervorragende Ergebnisse publiziert [9, 10]. Klassischerweise stellt die höhergradige Verletzung eine Operationsindikation dar. Individualentscheidungen sind v.a. im Bereich von RockwoodIII-Verletzungen zu treffen.

Im Rahmen dieses Beitrags können nicht alle Möglichkeiten der operativen Versorgung einer AC-Gelenkssprengung erörtert werden. Gängige und auch in unserer Klinik regelhaft angewandte Operationstechniken sind die KirschnerDraht-Fixation und die Versorgung mittels TightRope ${ }^{\circledR}$. Diese Verfahren, ihre Indikation sowie Tipps und Tricks werden im vorliegenden Artikel beschrieben. Weiter stellen wir unser Nachbehandlungskonzept der operierten Schulter vor und geben Tipps zur operativen Versorgung der AC-Gelenksarthrose.

\section{Anatomie}

Die Bedeutung des AC- und SC-Gelenks für die Beweglichkeit der Schulter ist eminent. Zwar fällt es schwer, die Beweglichkeit der einzelnen Gelenkkompartimente am Schulterblatt und Schlüsselbein klinisch zu quantifizieren, jedoch sind die beiden Schlüsselbeingelenke funktionell zu einem Drittel an der Hebebewegung des Armes beteiligt. Dabei entfallen $40 \%$ der Schulterblattbewegung auf das AC-Gelenk [11]. Bei der Betrachtung des von kranial-lateral nach kaudal-medial laufenden AC-Gelenk- 


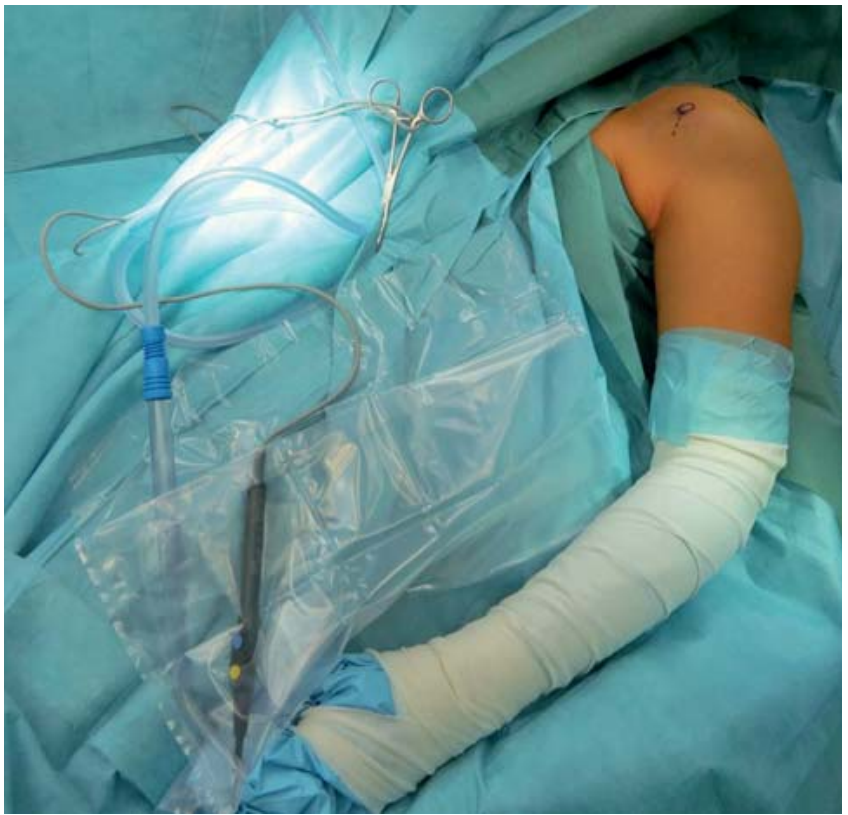

Abb. 1 Armlagerung: frei beweglich am Ellenbogen nicht aufliegend.

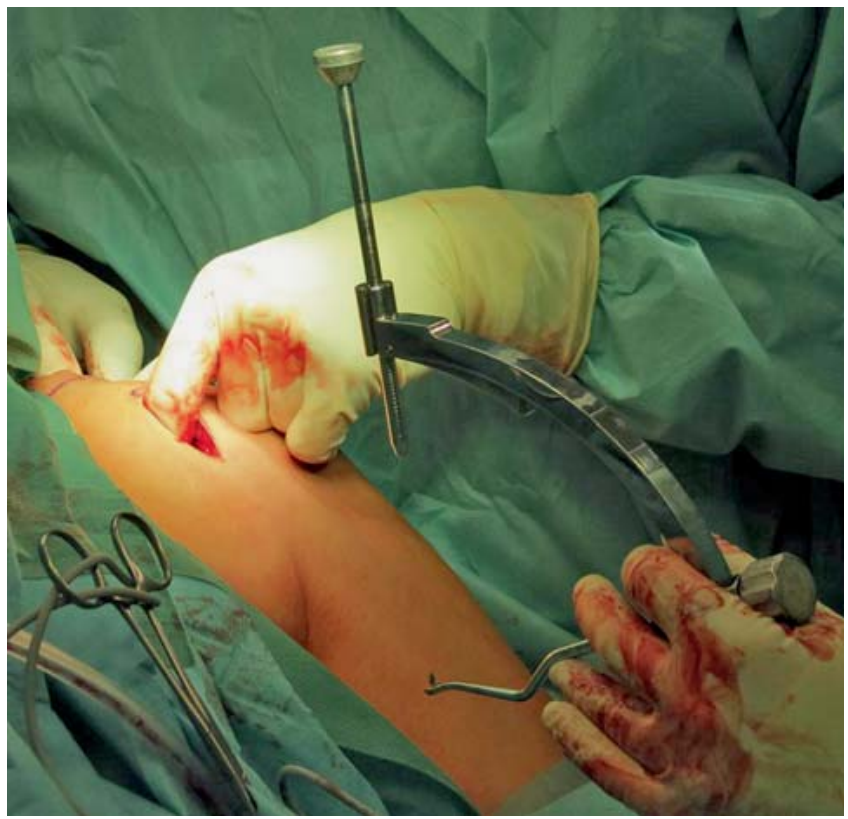

Abb. 2 Einbringen des Zielgeräts. spalts gibt es große individuelle Unterschiede. Eine Klassifikation erfolgt nach DePalma. Entsprechend der Funktion des AC-Gelenks als kraftübertragender Ort vom Rumpf auf den Schulterarmkomplex (bei Belastung auch entgegengesetzt) wird das Gelenk durch stabile Bandstrukturen, die korakoklavikulären Bänder, gesichert. Als horizontale Stabilisatoren werden das Lig. trapezoideum (posterior) und das Lig. conoideum (anterior) sowie die Muskelfaszien der $\mathrm{Mm}$. deltoideus und trapezius beansprucht. Zur weiteren Stabilisierung tragen der Discus interarticularis und die akromioklavikulären Bänder bei. Vertikal wird das Schultereckgelenk durch das Lig. conoideus und die genannten Muskelfaszien stabilisiert [12,13].

\section{Diagnostik}

Degenerative Beschwerden am AC-Gelenk werden durch den Betroffenen als Schmerz bei adduzierenden und extendierenden Bewegungen sowie dem Tragen schwerer Gegenstände am herunterhängenden Arm beschrieben. Schmerzen projizieren sich auf die Pars descendens des M. trapezius und den M. sternocleidomastoideus. In den klinischen Untersuchungen sind die ACGelenkprovokationstests positiv. So kann der O'Brien-Test, welcher ebenfalls für die Diagnose einer labralen Läsion spezifisch ist, auch für das AC-Gelenk Anwendung finden.
Die probeweise Infiltration mit einem Lokalanästhetikum dient als weitere Hilfe, das AC-Gelenk als Ort der Schmerzausstrahlung zu verifizieren. In der traumatischen Genese beschreibt der Patient oft ein direktes Anpralltrauma bei adduziertem Arm. Hier fallen Prellmarken und oftmals eine Fehlstellung der dann nach kranial luxierten lateralen Klavikula gegenüber dem Akromion auf.

In der apparativen Diagnostik kommen die a.-p. Aufnahme des Glenohumeralgelenks sowie die Y-Aufnahme oder die transaxilläre Aufnahme zur Geltung. Um den AC-Gelenkspalt einsehen zu können, muss der Strahlengang in a.-p. jedoch nach Zanca (1971) ansteigend etabliert werden. Mit der Alexander-Aufnahme gelingt der Nachweis von AC-Gelenk (sub)luxationen. Beim Verdacht einer Gelenkluxation erfolgt die Darstellung der AC-Gelenke im Seitenvergleich und unter Belastung. Zum Nachweis einer horizontalen Instabilität ist es essenziell, auch eine transaxilläre Aufnahme anzufertigen. Sonografisch lassen sich degenerative Veränderungen am AC-Gelenk gut darstellen. Auch sind sowohl niedrig klassifizierte frische Gelenksverletzungen (Rockwood I-II) bei entsprechender Anamnese durch den entstehenden Erguss wie auch höhergradige (Rockwood IV-VI) Verletzungen durch Muskelhämatome oder eingerissene Faszienansätze an der Klavikula diagnostizierbar. Im Seitenvergleich kann der korakoklavikuläre Abstand gut gemessen werden und korreliert mit dem Röntgenbild [14,15].
Weitere Möglichkeiten bieten je nach Indikation weniger die CT oder Szintigrafie (Arthrose) als die MRT. In der MRT zeigen sich v.a. schon früh Osteolysen, wie sie bei jungen Menschen mit starker Beanspruchung des AC-Gelenks auftreten, und rheumatische Veränderungen.

Auch detektiert die MRT bei frischen Luxationen eine Vielzahl ansonsten übersehener Begleitverletzungen.

So konnten Tischer et al. nachweisen, dass SLAP-Läsionen mit 15\%, Frakturen mit 5\% und Rotatorenmanschettenverletzungen mit $4 \%$ bei höhergradigen ACGelenkluxationen vorhanden sind [16]. Klassifikationen zur Beschreibung der AC-Gelenkluxation wurden durch Tossy (1963) und Rockwood (1984) erstellt. Kraus et al. stellten 2010 mit dem Acromioclavicular Joint Instability Score (ACJI) ein neues Evaluationswerkzeug für Schultereckgelenksinstabilitäten vor.

\section{Operative Therapie der frischen AC-Gelenkssprengung}

Bei der operativen Versorgung der ACGelenkssprengung müssen zerrissene, ggfs. nicht mehr rekonstruierbare Bandund Diskusstrukturen reseziert werden. Alsdann gehört die Klavikula korrekt in 2 Ebenen reponiert. Zur Sicherung des Alignments muss die Stellung fixiert und der Bandapparat so zur Ausheilung gebracht werden. Möglicherweise sind die Faszien des M. deltoideus oder M. trapezius eingerissen bzw. weitere 


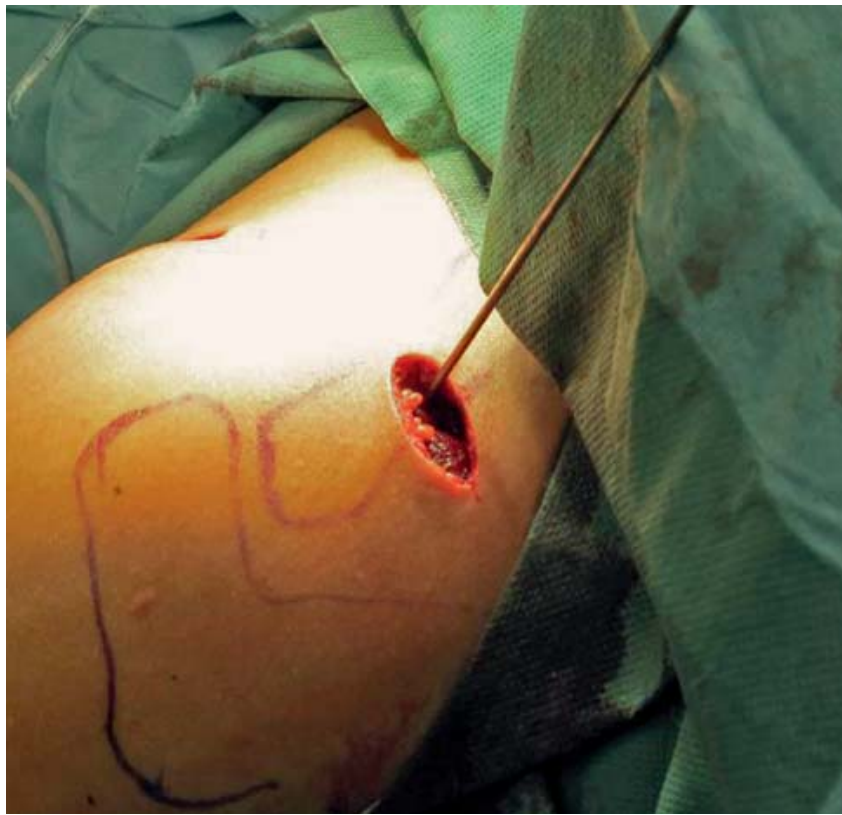

Abb. 3 Der K-Draht folgt dem Bandverlauf.

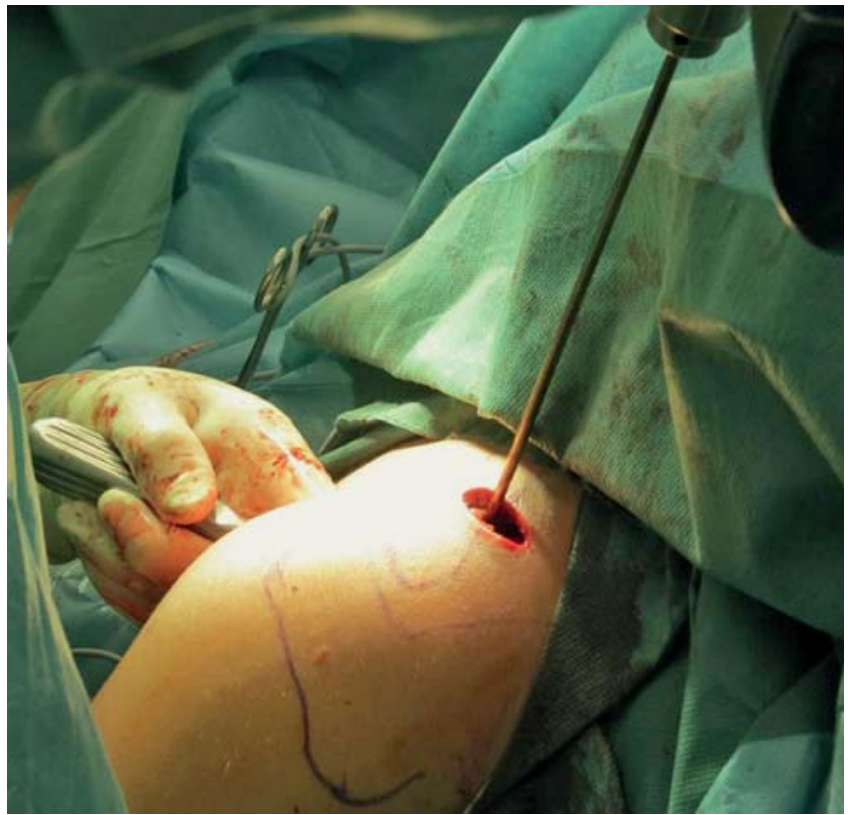

Abb. 4 Die Spitze des K-Drahtes wird beim Überbohren mit einem Löffel geschützt. anatomische Strukturen im Schulterbereich verletzt und müssen versorgt werden.

Vor allem hinsichtlich ihrer Bedeutung für die horizontale Stabilität ist eine Versorgung der Faszien im Bedarfsfall sehr wichtig.

\section{Lagerung}

Der Patient wird in Beach-Chair-Position gelagert und der Kopf in einer dafür vorgesehenen Kopfschale fixiert. Es sollte darauf geachtet werden, dass kein Metall den Strahlengang des C-Bogens stört. Im eigenen Vorgehen wird der Patient zur operierenden Seite hin einige Zentimeter über die Mittellinie des Tisches gelagert. Der Tisch wird dezent zur Gegenseite gekippt, um ein Abrutschen zu verhindern. Der Arm wird auf einer Armschiene gelagert, wobei das Ellenbogengelenk nicht aufliegt (Abb. 1). Abschließend werden die ventrale und dorsale Schulterpartie bis hin zum Sternoklavikulargelenk frei beweglich abgedeckt. Der Arm wird bis hin zum M. biceps brachii mit einer Stockinette abgedeckt.

\section{Welches Verfahren?}

Betrachtet man die Schulteranatomie und die verfügbaren Operationsverfahren, so kann man zwischen Verfahren wählen, die gelenkübergreifend (akromioklavikulär), und Techniken, die den
Processus coracoideus miteinbeziehen, wählen. Kombinationsverfahren beider Techniken sind ebenfalls möglich.

In der eigenen Klinik finden regelhaft 2 Techniken Anwendung: Dabei hat die Versorgung mittels TightRope ${ }^{\circledR}$-System (Fa. Arthrex, Naples/USA) gegenüber der transartikulären Fixation mittels Kirschner-Drähten an Bedeutung gewonnen. Murray und Phemister beschrieben erstmals die transartikuläre Fixation mittels Kirschner-Drähten. Technisch ist dieses Verfahren unkompliziert. Die Lagerung erfolgt wie beschrieben in Beach-ChairPosition. Über einen frontalen, parallel zur Klavikula und über das ACG verlaufenden Zugang wird von medial des Processus coracoideus bis ca. 1,5-2 cm über die laterale Akromionkante hinaus der Schnitt angelegt und unter sparsamer Präparation auf die Bandstrukturen zugegangen. Der Säbelhiebschnitt bietet sich ebenfalls an und reduziert das Risiko nervaler Schädigungen. Lig. acromioclaviculare, die korakoklavikulare und deltotrapezoidale Faszie wird einsehbar und die Eintrittsstelle der K-Drähte kann vorbereitet wurden.

In Erweiterung der reinen K-Draht-Fixation wird eine zusätzliche Cerclage in Achterform empfohlen. Vorteile entstehen durch eine höhere Stabilität und ein nur geringes Risiko der Drahtdislokation. Mithilfe der Zuggurtung kann die Gelenkspaltbreite dosiert werden.
Dies ist aber auch der größte Nachteil der Cerclagentechnik, denn durch übermäßiges Komprimieren der Gelenkkompartimente besteht die Gefahr der Knorpelschädigung und vorzeitigen degenerativen Veränderung.

Entscheidet man sich für die Anlage einer Cerclage, so wird das dafür notwendige Bohrloch mit einem 2-mmBohrer ca. $3 \mathrm{~cm}$ medial des ACG in der Klavikula angelegt. Zur gleichmäßigen Kräfteverteilung sollte auf eine Bohrlochrichtung in $90^{\circ}$ zur Klavikulaachse geachtet werden.

Nachdem nicht rekonstruierbare Bandanteile reseziert sind und die noch rekonstruierbaren Bandstümpfe mit einem resorbierbaren Faden armiert wurden, können vor der Reposition die Inspektion und das Débridement des Discus interarticularis erfolgen. Die Reposition wird dann mittels Spitz-Spitz-Zange gehalten und in 2 Ebenen radiologisch geprüft.

Alsdann erfolgt das Einbringen zweier Kirschner-Drähte mit je $2 \mathrm{~mm}$ Durchmesser. Diese verlaufen parallel und werden von der lateralen Akromionkante zunächst über das ACG in das laterale Klavikulaende eingebracht. Die Verankerung in der Gegenkortikalis ist essenziell. Mehrfache Bohrversuche sollten aufgrund der Knorpelschädigung unterlassen werden. Aus diesem Grund verwenden einige Autoren nur 1 KirschnerDraht. Die in Kauf genommene Rota- 


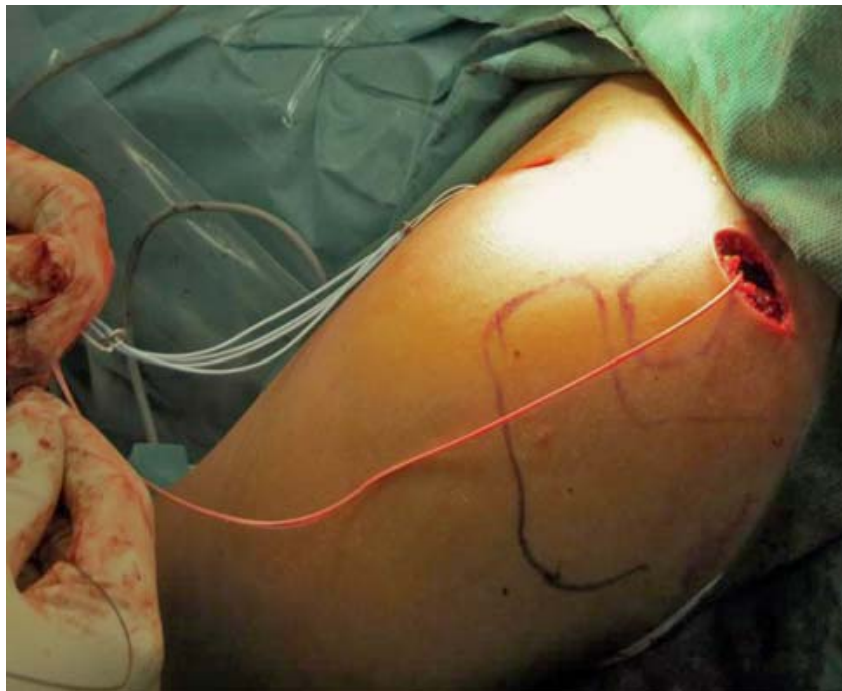

Abb. 5 Das TightRope ${ }^{\circledR}$ wird eingezogen.

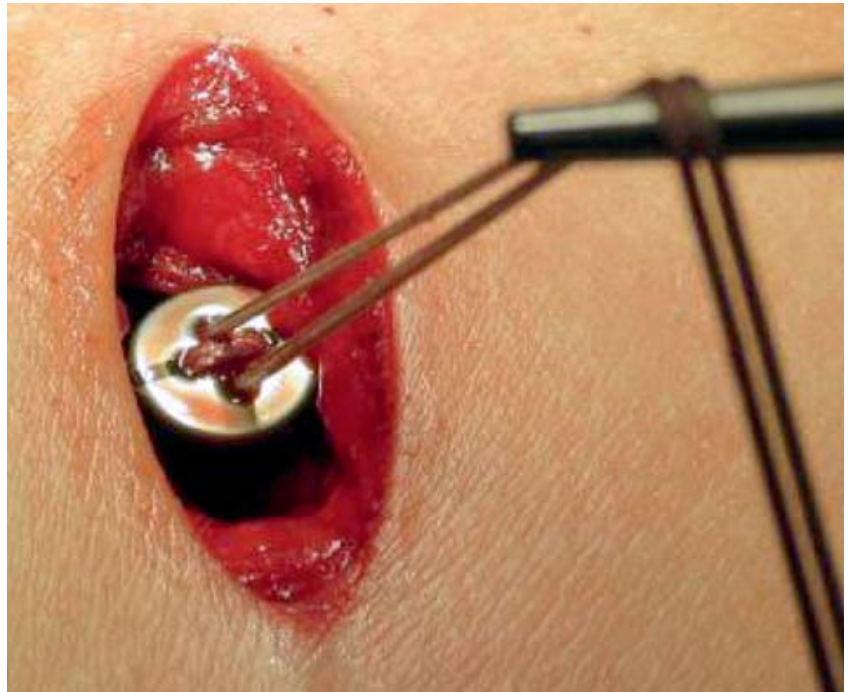

Abb. 6 Der Knoten-Button kommt unter dem Processus coracoideus zu liegen. tionsinstabilität erhöht jedoch das Risiko eines Materialbruchs oder der -lockerung, sodass im eigenen Vorgehen die Anlage zweier Drähte bevorzugt wird, auch wenn sich dadurch die Rigidität im ACG erhöht. Nachdem das Repositionsergebnis erneut und ohne Repositionszange radiologisch verifiziert wurde, wird nun der Cerclagendraht durchgezogen und in Achtertour unter die K-Drähte gebracht. Der Draht wird vorgespannt und das Drahtschloss angelegt. Die KDrähte werden umgebogen und versenkt. Abschließend werden die vorgelegten Nähte verknüpft und die ggfs. eingerissene Deltotrapezoidea transossär verknüpft.

Diese Technik findet ebenfalls Anwendung bei lateraler Klavikulafraktur mit ausreichend medial gelegener unverletzter klavikulärer Knochensubstanz, bei der eine Verankerung von K-Drähten noch problemlos möglich ist. Überschreitet die laterale Klavikulafrakturzone jedoch die korakoklavikulären Bandansätze, erschwert die Form des Schlüsselbeins diese Operationstechnik. Hier können Hakenplatten zum Einsatz kommen. Im eigenen Vorgehen verwenden wir die Hakenplatte bei isolierter ACGDislokation nicht. Zwar scheinen weiterentwickelte Hakenplatten (z.B. nach Dreithaler) nicht mehr die Nachteile früherer Modelle aufzuweisen, jedoch sind die Ergebnisse auch im Langzeitvergleich nicht überzeugend. KirschnerDrähte bieten sich auch bei begleitenden Frakturen des Processus coracoideus an, welche eine Versorgung der ACG-Sprengung mittels korakoklavikulärer Stabilisierung (Bandaugmentation oder Tight-
Rope $^{\circledR}$ ) verhindern. Nachteilig bei den vorbeschriebenen Verfahren ist die fehlende anatomische Rekonstruktion sowie die fehlende physiologische Biomechanik. Weiter ist die Zweitoperation zur Metallentfernung notwendig. Dies birgt Risiken für den Patienten und verursacht zusätzliche Kosten. Unter Umständen tritt sogar eine Reluxation des ACG auf. Die Notwendigkeit der Metallentfernung ergibt sich aus der eingeschränkten Mobilität sowie der Gefahr einer schon genannten Implantatwanderung. So fanden sich schon K-Drähte weit weg vom Ort der eigentlichen Implantation.

Eine Möglichkeit der korakoklavikulären Stabilisierung bietet hingegen die Versorgung mittels TightRope ${ }^{\circledR}$ (Fa. Arthrex, Naples/USA). Im Vergleich der Verfahren konnten wir nachweisen, dass ein gleichwertiges Repositionsergebnis erzielt wird. Signifikante Unterschiede zum Vorteil einer Verfahrenswahl gab es jedoch nicht [21]. Die OP-Technik kann arthroskopisch assistiert durchgeführt werden. Die Versorgung von Begleitverletzungen im Bereich der Faszien kann dann jedoch nicht erfolgen. Im eigenen Vorgehen favorisieren wir die Versorgung mittels TightRope ${ }^{\circledR}$ und legen entsprechende Miniinzisionen über der Klavikula und dem Processus coracoideus an. Diese Zugänge ermöglichen es, o.g. zusätzliche Verletzungen im Bereich der deltotrapezoidalen Faszie zu versorgen. Über einen Säbelhiebschnitt im Bereich der dorsalen Klavikula erfolgt die Präparation auf den Knochen, welcher hier mit einem stumpfen Raspatorium etwas weiter nach medial und lateral freigelegt wird. Das ACG wird nicht tangiert. Anschließend erfolgt die Schnittführung im Bereich des Processus coracoideus. Hier präpariert man bis auf die Bizepssehne. Diese wird in Längsrichtung gespalten, bevor man auf die Spitze des Processus coracoideus trifft. Stumpf wird von hier aus die Unterseite bis hin zur Basis und von dort aus weiter mit dem stumpfen Raspatorium die Basis des Korakoids ausreichend frei dargestellt. Als Nächstes erfolgt das Einbringen des Zielgeräts (Abb.2). Dieses wird unter der Basis des Korakoids positioniert und im Bereich der dorsalen Klavikula ausgerichtet. Mittels Kirschner-Draht werden die Klavikula und der Processus coracoideus durchbohrt (Abb. 3). Dies geschieht entlang des Bandverlaufs und sollte beim 1. Bohrloch das mittig unterhalb der Basis liegende Zielgerät treffen. Hier inseriert das Lig. conoideum. Nach korrekter Lagekontrolle erfolgt das Überbohren mit einem 4-mm-Bohrer. Mit einem scharfen Löffel sollte dabei die K-DrahtSpitze geschützt und so ein versehentlich akzidentelles Tiefertreten in die Weichteile verhindert werden (Abb.4). Nachdem der Draht erfolgreich überbohrt wurde, kann er entfernt werden, und ein Führungsdraht mit Schlaufe wird durch den kanülierten Bohrer geführt und mit der Fasszange unterhalb des Processus coracoideus gefasst und herausgezogen. Die Bohrhülse kann nun entfernt werden. Nun kann man je nach Wahl das 2. Bohrloch anlegen.

Ob die Bohrlöcher durch die Klavikula parallel laufen oder $\mathrm{V}$-förmig angelegt werden sollten, ist nicht abschließend geklärt. 


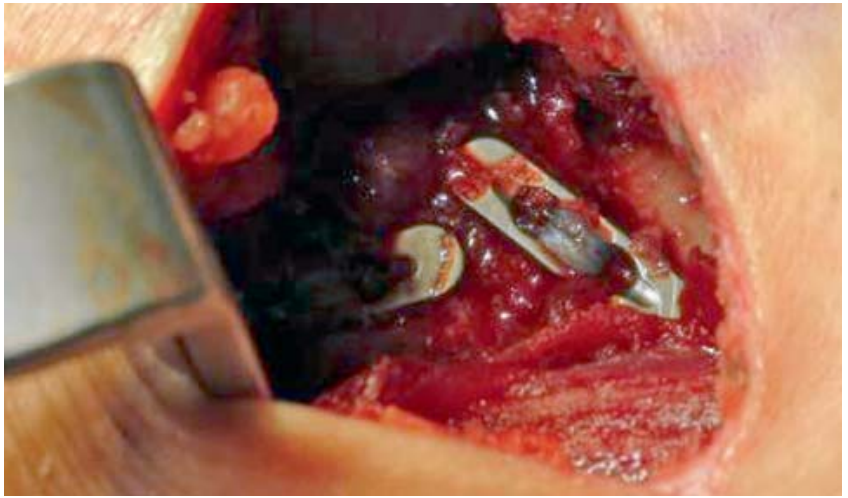

Abb. 7 Die Endo-Buttons sind umgeflippt und liegen der Klavikula auf.

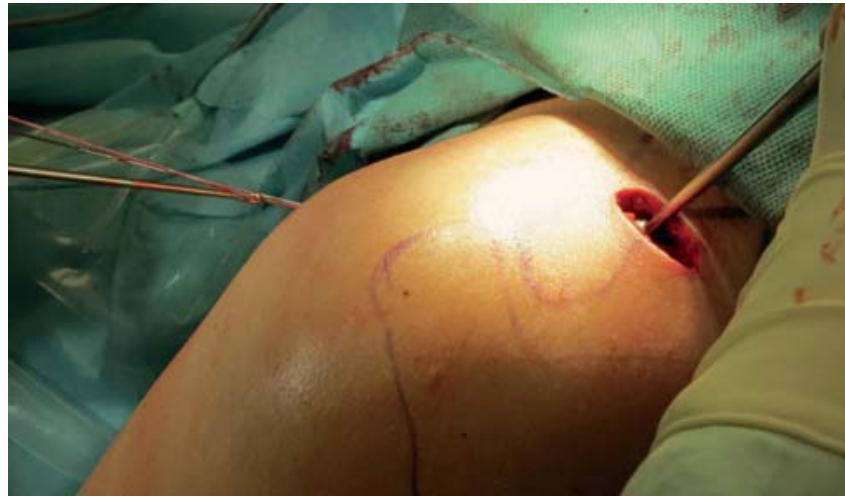

Abb. 8 Die Reposition erfolgt mit dem Kugelspieß - die Knoten werden mit dem Knotenschieber unter das Korakoid gelegt.
In einer ersten Nachuntersuchung von Kraus et al. konnten bei beiden Verfahren gute bis sehr gute Ergebnisse erzielt werden. Eine partielle vertikale und horizontale Restinstabilität war in beiden Gruppen zu beobachten [22]. Das 2. Bohrloch durch den Processus coracoideus sollte, gemäß des Bandansatzes des Lig. trapezoideum, $1 \mathrm{~cm}$ anterior des 1. Bohrlochs und $5 \mathrm{~mm}$ lateral der medialen Begrenzung des Processus coracoideus angelegt werden. Auch hier erfolgt die Überbohrung des K-Drahtes und Einbringen eines Führungsdrahts. Alternativ kann nach der 1 . Bohrung auch zunächst ein TightRope ${ }^{\circledR}$ in den Führungsdraht eingespannt werden. Dieses lässt sich problemlos zunächst durch die Klavikula und dann den Processus coracoideus ziehen (Abb.5). Der Endo-Button flippt auf der Unterseite des Processus um und der Knopf-Button kann problemlos auf der Dorsalseite der Klavikula positioniert werden. Sobald beide TightRopes eingezogen sind, erfolgt die Reposition und die Kontrolle des Repositionsergebnisses unter dem Bildwandler (Abb.9). Nun werden die TightRopes auf dem proximalen EndoButton verknotet. Dabei muss darauf geachtet werden, dass der Button dem Knochen anliegt und mögliche Weichteile nicht zwischen ihm und der Klavikula eingeklemmt werden. Weiter können die Enden des nach der Verknotung gekürzten Fadens zu Weichteilirritationen und Wundheilungsstörungen führen. Hier bietet es sich an, die Fäden ausreichend lang zu belassen oder gar auf der Vorderseite der Klavikula entlangzuführen, um eines der TightRopes zu schlingen und dann erneut zu verknoten, sodass diese Gefahr minimiert wird.
Im eigenen Vorgehen wechseln wir bei besonders schlanken Patienten und fehlenden Weichteilen oberhalb der Klavikula die Richtung beim Einbringen des TightRopes.

Das TightRope ${ }^{\circledR}$ wird dann von kaudal erst über den Processus coracoideus und dann durch die Klavikula geführt, sodass der Endo-Button auf der Klavikula umschlägt und der Knoten-Button auf der Unterseite des Processus coracoideus zu liegen kommt (Abb. 6 und 7). Mit einem Knotenschieber, wie er bei der arthroskopischen Bankart-Repair zum Einsatz kommt, lässt sich das TightRope ${ }^{\circledR}$ auf der Unterseite des Processus coracoideus gut verknoten (Abb.8). Die Weichteilirritationen im Bereich der Klavikula werden so verhindert. Ein sorgfältiger Wundverschluss bildet den Abschluss der Operation.

\section{Operative Therapie der AC-Gelenksarthrose}

\section{OP-Verfahren bei AC-Gelenksarthrose}

Die AC-Gelenksarthrose kann verschiedene Ursachen haben. Als Folge einer früheren AC-Gelenkssprengung kann sie auftreten. Sollten konservative Verfahren in der Behandlung der AC-Gelenksarthrose versagen, so stehen unterschiedliche operative Verfahren zur Wahl. So beschrieben Gurd und Mumford 1941 die offene Gelenkresektionsplastik, bei der durch einen entsprechenden Zugangsweg das AC-Gelenk freigelegt wird. Wichtig ist hier, die Kapsel in horizontaler Richtung zu spalten und den Gelenkspalt freizulegen. Anschließend erfolgen die Resektion der lateralen Klavikula mit der oszillierenden Säge und die Entfernung des Discus interarticularis. Die Studienlage bez. der Resektions- strecke ist reichhaltig und reicht von ca. $2,5 \mathrm{~cm}$ über $1,8 \mathrm{~cm}$ bis hin $\mathrm{zu} 1 \mathrm{~cm}$.

Insgesamt muss festgehalten werden, dass eine weite Resektionsstrecke über $1 \mathrm{~cm}$ mit einem schlechteren Outcome assoziiert zu sein scheint.

Dies liegt vermutlich daran, dass mit Überschreiten dieser Distanz die korakoklavikulären Bandansätze in Mitleidenschaft gezogen werden, sodass deren Funktion beeinträchtigt wird $[17,18]$. Die Resektion der lateralen Klavikula kann auch über den bekannten subakromialen Zugang mittels Shavern erfolgen. Hierbei werden die korakoakromialen Bänder jedoch teilreseziert. Ein ebenfalls endoskopisch gangbarer Weg ist der der intraartikulären Resektion mittels MiniShavern. $\mathrm{Zu}$ beachten sind jedoch der größere technische Aufwand, die mit dem besonderen Instrumentarium verbundenen Kosten und die für den Ungeübten anspruchsvolle Technik. Letztlich muss darauf geachtet werden, dass bei ausreichender Beweglichkeit des Armes die Gelenkkompartimente nicht aneinander reiben, was postoperativ $\mathrm{zu}$ Schmerzen für den Patienten führt. Auch ist peinlichst auf Schonung der korakoklavikulären Bandstrukturen zu achten. Eine Rekonstruktion ist schwierig.

Nach Weaver-Dunn (1972) werden Teile des Bandapparats in die Operationstechnik von Gurd/Mumford eingebunden. Das Lig. coracoacromiale wird nach der Resektion der lateralen Klavikula von der Unterseite des Processus coracoideus zusammen mit einer Knochenlamelle abgelöst und am lateralen Ende der Klavikula mittels Nähten befestigt. Technisch ist die Operationstechnik einfach und ermöglicht zudem die Versorgung von Begleitverletzungen in diesem Be- 


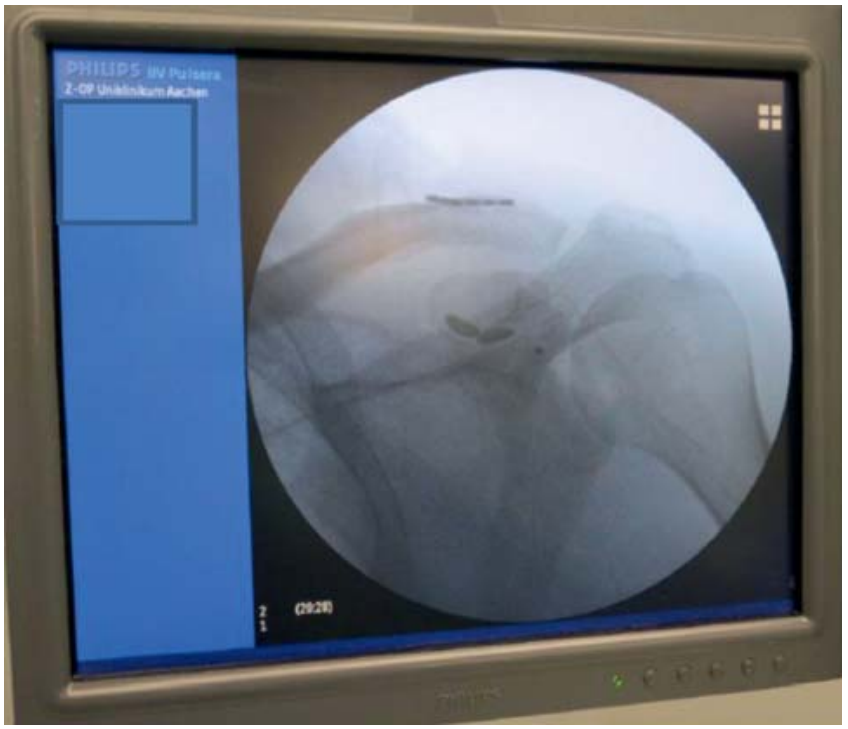

Abb. 9 Radiologische Kontrolle.

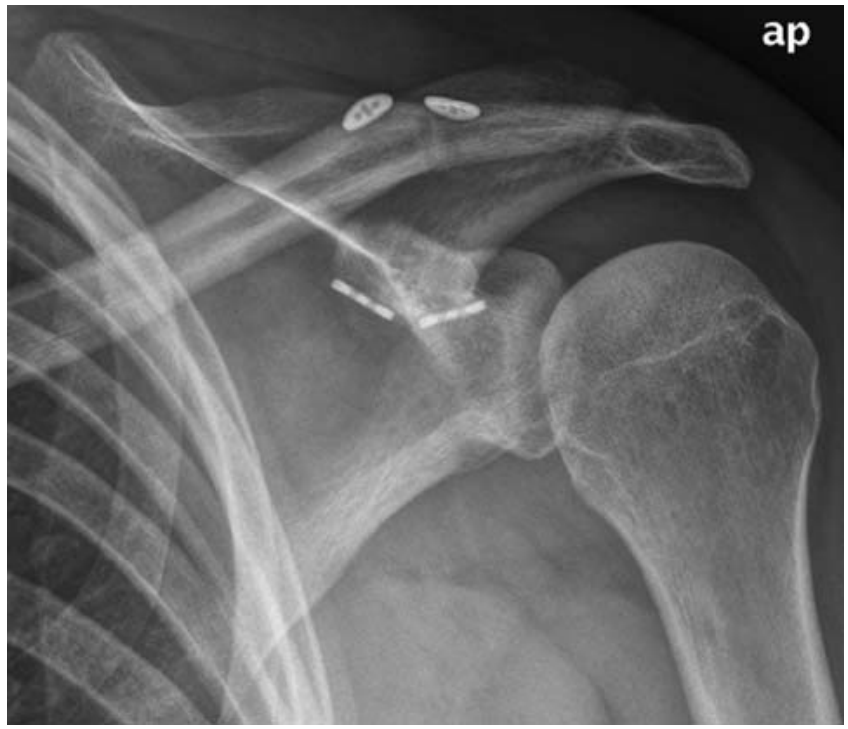

Abb. 10 3-Monats-Ergebnis nach Double-TightRope ${ }^{\circledR}$ (DTR). reich. Andererseits muss bedacht werden, dass gerade bei der Behandlung veralteter AC-Gelenkssprengungen die horizontale Stabilität mit diesem Verfahren nicht ausreichend adressiert wird und ein höhergradiger vertikaler Versatz der lateralen Klavikula zusätzlich mit einer Bandaugmentation versorgt gehört.

Bei horizontaler Instabilität muss eine Rekonstruktion der anatomischen Verhältnisse angestrebt werden.

Hier bieten sich Sehnenersatzverfahren an, welche sich in ersten biomechanischen Studien Erfolg versprechend zeigen $[19,20]$. Es gilt, dass die voroperierte Schulter, welche vielleicht schon einer Weaver-Dunn-Operation oder einer Sehnentransplantatoperation unterzogen wurde, in ihrer Anatomie so verändert ist, dass sie nicht in die Hand des unerfahrenen Operateurs gehört. So müssen der Revisionseingriff und die Sehnenersatzentnahmestellen gründlich vorbereitet und der Patient in der Nachbehandlung eng begleitet werden.

\section{Nachbehandlung}

Dem Patienten wird noch im Operationssaal ein Gilchrist-Verband angelegt. Wir empfehlen das Tragen des Verbands über den Zeitpunkt der Wundheilung hinaus für mindestens 2, besser 3 Wochen. $\mathrm{Zu}$ frühe Belastung führt ggfs. zu ausbleibender Heilung im Bereich der deltotrapezoidalen Insertionen. Ab der 1. postoperativen Woche wird mit passiver assistierter Bewegung begonnen. Limitationen liegen in der Flexion und Abduk-

Tab. 1 Nachbehandlungsschemata bei Versorgung mittels K-Drähten und TightRope ${ }^{\circledR}$.

\begin{tabular}{|c|c|c|}
\hline Postoperative Woche & K-Draht & TightRope \\
\hline 1.-2. & \multicolumn{2}{|c|}{$\begin{array}{c}\text { passiv } \\
\text { IRO/ARO 90-0-0 } \\
\text { Abd./Flex. max. } 30^{\circ}\end{array}$} \\
\hline 3.-4. & $\begin{array}{l}\text { aktiv-assistiert } \\
\text { IRO/ARO } 90-0-0 \\
\text { Abd. max. } 70^{\circ} \\
\text { Flex. max. } 90^{\circ}\end{array}$ & $\begin{array}{l}\text { aktiv-assistiert } \\
\text { IRO/ARO 90-0-0 } \\
\text { Abd./Flex. max. } 45^{\circ}\end{array}$ \\
\hline 5.-6. & \multicolumn{2}{|c|}{$\begin{array}{c}\text { aktiv } \\
\text { IRO/ARO frei } \\
\text { Abd./Flex. max. } 60^{\circ}\end{array}$} \\
\hline 7.-8. & Entfernen der K-Drähte & freie Beweglichkeit \\
\hline 12.-16. & vol & \\
\hline$(16 .-24)$. & Aufbauphase für Spitzer & n volle Belastung \\
\hline
\end{tabular}

tion bei $30^{\circ}$, und die Außenrotation sollte nicht über die Null-Grad-Ebene erfolgen. Ab der 3. Woche kann mit aktiv-assistierter Beübung begonnen werden. Abduktion und Flexion sollten $45^{\circ}$ nicht überschreiten und eine Außenrotation ist ebenfalls bis zum Abschluss der 4. postoperativen Woche zu vermeiden.

Bei der Versorgung mittels K-Drähten sind Limitationen von $90^{\circ}$ in der Flexion und $70^{\circ}$ in der Abduktion einzuhalten, um das einliegende Material nicht zu überfordern und den Materialbruch zu provozieren.

Bei der Versorgung mittels TightRope ${ }^{\circledR}$ wird ab der 5. Woche die Außenrotation freigegeben und die Limitation bei Flexion und Abduktion auf $60^{\circ}$ erhöht sowie ab der 7 . Woche der Patient ganz freigegeben. Die Freigabe erfolgt auch für den mit K-Drähten versorgten $\mathrm{Pa}$ tienten nach Entfernung der Drähte zu diesem Zeitpunkt. Eine volle Belastbarkeit ist ab dem 3.-4. Monat gegeben (Tab. 1, Abb. 10).

\section{Literatur}

${ }^{1}$ Graupe F, Dauer U, Eyssel $M$. [Late results of surgical treatment of Tossy III acromioclavicular joint separation with the Balser plate]. Unfallchirurg 1995; 98: 422-426

2 Thielke KB, Barth F, Echtermeyer V. Akromioklavikulargelenkverletzungen: Operative Möglichkeiten und Resultate. Trauma Berufskrankh 2004; 6: S334-S338

3 Rockwood C-J, Madsen III FA. The Shoulder, Vol 1. Philadelphia: Saunders; 1990

${ }^{4}$ Lizaur A, Sanz-Reig J, Gonzalez-Parreno S. Long-term results of the surgical treatment of type III acromioclavicular dislocations: an update of a previous report. J Bone Joint Surg [Br] 2011; 93: 1088-1092 
${ }^{5}$ Dumrongwanich P, Piyapittayanum P. Outcomes of percutaneous K-wire fixation for AC joint separation type III. J Med Assoc Tha 2009; 92 (Suppl. 6): S211-S216

${ }^{6}$ Chen SK, Chou PP, Cheng YM, Lin SY. Surgical treatment of complete acromioclavicular separations. Kaohsiung J Med Sci 1997; 13: 175-181

${ }^{7}$ Petersen W, Wellmann M, Rosslenbroich S, Zantop T. [Minimally Invasive Acromioclavicular Joint Reconstruction (MINAR)]. Oper Orthop Traumatol 2010; 22: 52-61

${ }^{8}$ Bektaser B, Bozkurt M, Ocguder A, Solak S, Oguz T. [Surgical treatment of type III acromioclavicular joint dislocations by a modified Bosworth technique]. Ulus Travma Acil Cerrahi Derg 2004; 10: 245-249

${ }^{9}$ Bathis H, Tingart M, Bouillon B, Tiling T. [Conservative or surgical therapy of acromioclavicular joint injury-what is reliable? A systematic analysis of the literature using "evidence-based medicine" criteria]. Chirurg 2000; 71: 1082-1089

10 Smith TO, Chester R, Pearse EO, Hing CB. Operative versus non-operative management following Rockwood grade III acromioclavicular separation: a meta-analysis of the current evidence base. J Orthop Traumatol 2011; 12 : 19-27

${ }^{11}$ Rockwood CA, Young DC. Disorders of the acromioclavicular Joint. In: Rockwood CA Matsen FA, eds. The Shoulder, Vol. 1. Philadelphia: Saunders; 1990

12 Fukuda K, Craig EV, An KN, Cofield RH, Chao $E Y$. Biomechanical study of the ligamentous system of the acromioclavicular joint. J Bone Joint Surg [Am] 1986; 68: 434-440
${ }^{13}$ Debski RE, Parsons 3rd IM, Fenwick J, Vangura A. Ligament mechanics during three degreeof-freedom motion at the acromioclavicular joint. Ann Biomed Eng 2000; 28: 612-618

14 Kock HJ, Jurgens C, Hanke J, Schmit-Neuerburg $K P$. [Standardized ultrasound examination for classification of instability of the acromioclavicular joint]. Unfallchirurgie 1994; 20: 66-71

${ }^{15}$ Matter HP, Gruber G, Harland U. [Possibilities of ultrasound diagnosis in Tossy type III acromioclavicular joint injuries in comparison with loaded roentgen images]. Sportverletz Sportschaden 1995; 9: 14-20

16 Tischer T, Salzmann GM, El-Azab H, Vogt S, Imhoff $A B$. Incidence of associated injuries with acute acromioclavicular joint dislocations types III through V. Am J Sports Med 2009; 37: 136-139

17 Boehm TD, Kirschner S, Fischer A, Gohlke F. The relation of the coracoclavicular ligament insertion to the acromioclavicular joint: a cadaver study of relevance to lateral clavicle resection. Acta Orthop Scand 2003; 74: 718721

${ }^{18}$ Eskola A, Santavirta S, Viljakka HT, Wirta J, Partio TE, Hoikka V. The results of operative resection of the lateral end of the clavicle. J Bone Joint Surg [Am] 1996; 78: 584-587

19 Costic RS, Labriola JE, Rodosky MW, Debski RE. Biomechanical rationale for development of anatomical reconstructions of coracoclavicular ligaments after complete acromioclavicular joint dislocations. Am J Sports Med 2004; 32: 1929-1936

${ }^{20}$ Mazzocca AD, Santangelo SA, Johnson ST, Rios CG, Dumonski ML, Arciero RA. A biomechanical evaluation of an anatomical coracoclavic- ular ligament reconstruction. Am J Sports Med 2006; 34: 236-246

${ }^{21}$ Horst K, Dienstknecht T, Pishnamaz M, Sellei RM, Pape HC. Radiologisches follow-up nach minimal invasiver ACG-Stabilisierung mittels TightRope ${ }^{\circledR}$-System. Deutscher Kongress für Orthopädie und Unfallchirurgie (DKOU 2012), Berlin, 23.-26.10.2012

${ }^{22}$ Kraus NG, Gerhardt C, Haas, NP, Scheibel M. Arthroskopisch-assistierte Schultereckgelenksstabilisierung in korakoklavikulärer Doppel-Tight-Rope Technik - Vergleich Vförmige versus parallele Bohrkanalorientierung. Deutscher Kongress für Orthopädie und Unfallchirurgie (DKOU 2012), Berlin, 23. -26.10 .2012$

\section{Dr. med. Klemens Horst}

Arzt in Weiterbildung

Dr. med. Thomas Dienstknecht

Funktionsoberarzt

Univ.-Prof. Dr. med. Hans Christoph

Pape, FACS

Direktor der Klinik

Klinik für Unfall- und

Wiederherstellungschirurgie

Universitätsklinikum der

RWTH Aachen

Paulwelsstraße 30

52074 Aachen

khorst@ukaachen.de 